\title{
ENRICHMENT METHODS IN TRACE ANALYSIS
}

\author{
Robert A. Chalmers \\ Chemistry Department, University of Aberdeen, Old Aberdeen, \\ Scotland
}

\begin{abstract}
Enrichment methods are classified according to the type of sample for which they are to be used, and basic analytical principles are applied to develop criteria for choice of enrichment method and conditions to be used.
\end{abstract}

The topic of 'enrichment' or 'preconcentration' of trace elements has assumed great importance not only in the field of high-purity materials but also in connection with pollution. Whatever the merits or demerits of the case against increasing pollution of the environment, the analyst is faced with the reality of having to try to determine traces of a wide variety of substances. One of the ironic consequences of the anti-pollution campaign is that it would be quite possible for legislation to be enacted setting an upper limit for the permissible concentration of a pollutant, and for that limit to be below the limits of detection for that substance. However, if that were the case, the prosecution would have a hard task to prove that the pollutant was present in concentrations above the permitted limit but below the detection limit (unless, of course, they were aware of Professor Liteanu's mathematical methods for dealing with such a situation ${ }^{1}$ ).

The subject of this paper was dealt with by Minczewski ${ }^{2}$ at the IUPAC Congress in Moscow in 1965, and by Mizuike ${ }^{3}$ in a book on physical methods of trace analysis. It is also the subject of a review that will appear in Talanta ${ }^{4}$. My purpose will therefore not be to give a review of applications, but rather to try to apply simple analytical ideas to the problem and to suggest lines of work. Of necessity, there will be some common ground between this paper and those already mentioned.

\section{GENERAL CONSIDERATIONS}

Basically there are two situations in which enrichment will be required, their common feature being that the species to be concentrated before its determination (the determinand) is present in amounts or concentrations 
too low to be determined directly. We may have a very dilute solution of all the components of the system, which can then all be regarded as trace components, or we may have a trace of determinand in a matrix consisting of large amounts of other species. Examples of the first type are lake or river waters, or polluted air. The second kind includes geological samples, ultra-pure materials, industrial process streams, and alloys. In both cases it is necessary to enrich the system in determinand, either by separation and concentration of the determinand itself or by the removal of all or part of the major components of the system. The choice of method will depend on the chemical composition of the sample and on the method of determination to be used. For example, atomic absorption spectrometry is often regarded as being highly selective, so it might be thought that a simple concentration step might enrich the system sufficiently for a direct determination to be made. Unfortunately such a step would also greatly increase the concentration of other species present, and it is well known that serious errors can be caused by high salt concentrations in atomic absorption methods, partly because of light-scattering by clotlets in the flame, and partly by the asymmetric Lorentz broadening of the spectral lines when there is a high concentration of other species present, and it is well known that serious errors can be volatilization of the determinand may not be a very useful way of separating and concentrating it. The point in the analysis at which enrichment is attempted may also depend on the method of determination. In activation methods, for example, enrichment and separation may be necessary before irradiation, in order to avoid high levels of high-activity isotopes such as ${ }^{24} \mathrm{Na}$ from sea-water.

Several problems are common to all enrichment methods. The most serious are those of contamination and loss. Whenever chemicals have to be used in the separation process (or concentration step) there is risk of introduction of determinand present as impurity in the reagents used, and the larger the amount of reagent needed, the bigger the risk and level of contamination. The amount of reagent needed is in turn dictated by the sample size, which itself is determined by the nature of the sample, the sensitivity of the method of determination, and sometimes by cost or scarcity. Ease of handling also plays a part in choosing the sample size. Solutions may generally be expected to be fairly homogeneous in composition, but solid materials may have severe segregation of impurities or trace constituents, and then the smaller the sample the greater the chance of its not being representative of the parent material. Examples arise from the use of atomic absorption methods as a tool in mineral prospecting and analysis for pollutants. On account of the sensitivity of the method a small sample is adequate for an analysis and in the case of nickel ores an injudicious choice of sample could lead to an apparently abnormally high nickel content and high hopes for shareholders. An injudicious choice of sample recently led to fears of mercury poisoning from oysters-the samples chosen came from a bed very near the effluent discharge from a battery factory, and the oysters from the commercial supply beds were found to have acceptable levels of mercury in them. The question of cost and scarcity is best exemplified by samples of moon rock, but examples nearer home are some of the high purity metals. No-one is going to use the whole of the available material for an analysis and have 
none left, and sample size may be restricted for that reason. Handling is illustrated by the early methods for determining cadmium in zinc-base diecast alloys by precipitation separation of 0.001 per cent cadmium from 96 per cent zinc, which required about $1 \mathrm{~kg}$ of sample, and the polarographic method requiring only $1 \mathrm{~g}$. Large samples are not only unwieldy but, as already said, need large amounts of reagents.

Rather a ridiculous situation arises if the chemical operations introduce amounts of determinand vastly in excess of those originally present, and at least one example is known of a cooperative survey for a particular trace element giving remarkable agreement because all the investigators had used the same method and same impure reagent, and so had determined the trace element in the reagent and not in the original sample. Blank determinations are of little use in such circumstances, since the statistical variation in the blank may be greater than the amount being sought. The only answer is to purify the reagents by application of separation methods (preferably the ones chosen for the enrichment) but this adds to the length and cost of the analysis. Contamination can also arise from airborne matter in the laboratory atmosphere, and special precautions may have to be taken to avoid this source of error. Care must be taken to avoid contamination from the tools used in preparation of the sample.

Loss of determinand can occur at all stages of the enrichment procedure, so it is desirable to use as few steps as possible. When the sample is a solution a possible source of loss is adsorption on the walls of the sample container. This loss is likely to be more severe with increasing dilution of the sample. It would be expected that samples such as sea-water would suffer less loss, on account of the greater competition for adsorption sites by the major species present, unless there were some specific chemical interaction with the container. Adsorption effects are best studied by use of radioisotopes, and a systematic study would be most useful. Most of the information is scattered through the literature, usually in papers on other topics, but a useful summary is given by Gorsuch ${ }^{5}$, who also deals with interaction of reagents with apparatus.

Loss may also occur by volatilization or thermal decomposition of a species, though this may turn out to be an advantage if the effect is quantitative and the material produced can be collected and/or determined. Loss may also occur if an additional extraction or precipitation step is necessary before the one intended for enrichment of the determinand. The chemistry of the system must be taken into account when a dissolution procedure is required, and conditions chosen so that no loss of volatiles occurs. A more insidious source of trouble and of apparent loss is the formation of inert complexes of the determinand, since the species of interest will then not display its normal chemical behaviour. A fairly recently discovered source of errors of this nature is the existence of mixed-cation bridged complexes, such as the copper(II)-citrate-chromium(III) ${ }^{6}$, uranyl-citrate-aluminium ${ }^{7}$ and copper(II)tartrate-aluminium ${ }^{8}$ complexes. These species are all kinetically inert, and their hidden danger for trace analysis is not only that one of the cations may appear to be absent when it is present, but also that it can appear to be present (if its amount is greater than that stoichiometrically equivalent to the other) but the amount found will be lower than the true value. 


\section{ENRICHMENT METHODS}

\section{Physical methods}

The most obvious methods of concentration are evaporation of solvent or of major components of the matrix if the sample is solid. Subject to the provisos about volatility and thermal stability of the determinand, the only problem is likely to be from interaction with the apparatus. If the determinand is volatile or thermally labile, freeze drying may provide the answer. Nonvolatile thermally unstable compounds may be dealt with by vacuum distillation. Vacuum distillation may also be useful for extraction of volatile traces from a non-volatile matrix, as in the vacuum-fusion methods for gases dissolved or trapped in solids.

Volatilization is often useful in activation methods, since non-active isotope can be added as carrier after the irradiation. One of the early successes of this technique was the determination of down to $10^{-9} \mathrm{~g}$ of arsenic ${ }^{9}$. The volatility of mercury has also been used to advantage, but a consequence of it is the loss of mercury from dilute standard solutions ${ }^{10}$. Some platinum metal oxides are volatile and can be collected. Sublimation of matrix material is often possible, e.g. of ammonium salts, and of tin as $\mathrm{SnI}_{4}$.

Filtration may be used for collecting particulate matter from liquids and gases, and extracts from the filter may then be analysed by suitable methods, e.g. the fluorimetric analysis of airborne particulate matter ${ }^{11}$. Dialysis may serve to remove large amounts of simple electrolytes such as sodium chloride, but the water must be carefully purified (or dialysed) first.

Ashing of biological and organic samples may be achieved either by aerial oxidation at elevated temperatures or by chemical interaction. Care must be taken, however, to avoid loss of volatile material and to avoid loss or gain by interaction with the apparatus. This topic has been thoroughly investigated by Gorsuch ${ }^{5}$.

\section{Chromatography}

Ion-exchange, partition, adsorption, thin-layer and gel-permeation chromatography have all beeen used for concentration of trace species. At first sight they are most attractive methods because they permit separation of species as well as enrichment. There are some problems associated with their use, however. Some elements are very strongly retained and cannot conveniently be eluted chemically. In that case it may be necessary to ash the chromatographic material (if it is organic) and recover the determinand from the ash, but then the ashing must be done properly, and the method is rather wasteful. If elution is incomplete there will be cross-contamination between successive samples. The chromatographic system may interact with the sample or cause contamination by yielding soluble or particulate decomposition products.

One advantage of paper chromatography, thin-layer chromatography, and ion-exchange membranes is that the chromatographic substrate can be irradiated after the enrichment step, and the activity measured. Sometimes enrichment techniques are used in combination. For example, copper has been collected on an ion-exchange membrane and then electroplated on to a polished platinum surface and measured by microprobe ${ }^{12}$.

There are essentially two ways of using chromatographic methods for 
removing the trace species or for removing the matrix elements. The first of these is the more useful (if the traces can be removed easily) because the loading capacity of the stationary phase will be very much greater than the amount of trace species present, so very large samples can be used, with an attendant increase in sensitivity. If the matrix species are to be removed on the stationary phase the sample size is limited by the loading capacity. Moreover, if a large volume of solution is used, the effluent will probably still have to be concentrated.

In work with organic species it must be remembered that large volumes of solvent may be used on chromatographic columns, and even small amounts of impurities in the solvent may cause severe contamination problems.

\section{Solvent extraction}

One of the main attractions about solvent extraction as a means of enrichment is that it may be possible to use the colour of the final extract as a means of determination. Because of the practical limitations on molar absorptivity (according to theory the maximum value attainable is about $10^{4}$ 1.mole ${ }^{-1} \cdot \mathrm{mm}^{-1}$ ) the sensitivity of spectrophotometric methods is not particularly high, and a considerable degree of enrichment may be necessary to make the method useful. It is customary to state that as the concentration of solute decreases, the efficiency of extraction also decreases, and so it becomes more difficult to obtain satisfactory recoveries and enrichment factors. This statement is quite true as it stands, but it needs qualifying by addition of the words 'if only the one solute is extracted'. It seems to me (I have not yet tested it in practice) that there may be a simple answer to this problem. The reason for the loss in efficiency is presumably simply that if we consider the formation of a neutral species by chelate formation between a metal ion and an anionic complexing agent, or by ion-association, then at very high dilution the degree of dissociation will be too high for complete extraction of the species. Now suppose we add another metal ion of similar charge and radius to the determinand ion, and form with the extracting agent a similar complex of similar stability under the same conditions of $\mathrm{pH}$ etc. The reagent ions are presumably unable to distinguish the two types of cation and will simply react with whichever one they happen to meet in the solution (a parallel exists for precipitation reactions, where zirconium and hafnium behave indistinguishably, and potassium and ammonium ions react with the same reagents). If such a situation exists and the solution is homogeneous, the ratio of the two species in the extract should be the same as that for what remains in the aqueous phase, and be the same as the initial ratio of the two species. The recovery for the trace component should then be the same as the recovery of the added species. It would be interesting to test this.

Given a satisfactory degree of extraction, however, it is possible to achieve a series of concentration steps by successive extraction and stripping reactions applied alternately. A concentration factor of ten at each stage would soon give an appreciable enrichment factor. Organic solvents are fairly volatile, and further enrichment can be achieved by leaving the solvent to evaporate at room temperature (with protection from airborne contamination, of course). The extract may also be dealt with by chromatographic fractionation 
or concentration, or irradiated, or sprayed directly into the flame for atomic absorption measurements.

One useful application of solvent extraction methods is that a non-specific reagent, such as dithizone or oxine, may be used to extract a group of metals which may then be differentiated and determined by atomic absorption, activation analysis etc. The possibility also exists of applying substoichiometric methods ${ }^{13}$. Sequential analysis for many elements is possible, in theory at any rate, by application of such group reagents in proper order, as proposed earlier ${ }^{14-16}$. An obvious drawback is that conditions of $\mathrm{pH}$ usually have to be adjusted, but it should be possible to work out a sequential scheme in which all the group reagents are used at the same $\mathrm{pH}$, so that an initial buffering would suffice for the whole analysis; the buffer solution would, of course, first have been treated by the whole set of reagents in order to remove metal impurities.

Solvent extraction may be used, of course, to remove the matrix species, and the development of the so-called liquid ion-exchangers has made this an attractive approach. Extraction is also applicable to organic and biochemical systems, and metal ions may be added to form extractable complexes.

Polarographic methods may also be included under the heading of solvent extraction, the mercury being the solvent. The use of the hanging drop mercury electrode followed by stripping voltammetry provides an elegant example of a concentration technique accompanied by a means of determination. Removal of trace or matrix components by electrolysis with a mercury pool cathode is also an example.

' Solvent extraction methods form the subject of a recent monograph ${ }^{17}$.

\section{Precipitation}

Co-precipitation and similar phenomena have long been a source of difficulty to the analyst, but as so often happens, a disadvantage may be turned into an advantage in certain circumstances, and co-precipitation is a common means of scavenging traces of elements from solution. Here again there is the problem of efficiency of collection, and again it seems to me that intelligent design of the system should give a satisfactory recovery. The arguments just used in connection with solvent extraction should apply here also, and joint precipitation of two similar species, one present in large excess relative to the other, should result in virtually complete recovery of the trace component. Note that here we prefer to speak of joint precipitation, to distinguish the method from co-precipitation, by which is normally meant precipitation of a species that is normally soluble under the conditions used.

If it is decided to use co-precipitation methods, then obviously the procedure should be designed so as to maximize the contamination of the carrier precipitate, and concentrated reagent solutions should be used with rapid mixing at room temperature. Similarly the carrier precipitate should be chosen for its known tendency to collect impurities from solution, and the adsorption isotherm should be of the Langmuir type, favouring high adsorption even at low concentration. There is probably enough information in the literature for selection of carriers to be fairly easy. Sometimes it is advantageous to arrange precipitation of more than one type of insoluble 
material; an ionic precipitate and a flocculated colloid may be more efficient than either alone.

Because of the risk of loss of determinand by co-precipitation, it is not usual to achieve enrichment by precipitation of the matrix elements, and when it is done, extra care must be taken on this account. It may sometimes be possible to remove matrix elements by controlled-potential electrolysis.

The reverse of precipitation-selective dissolution-may also be used for enrichment. Finely divided solid sample is treated with a solvent that will dissolve the trace species to a much greater extent than it will the matrix species. The dissolution may be assisted by application of ultrasonic waves ${ }^{18}$. One of the difficulties in research on this method is that of obtaining standard materials to work with. It is very difficult to introduce impurity uniformly into a solid material, and to know how much has been introduced. Solutions present fewer problems, the major one being loss by adsorption. Preparation of solid samples of contaminated salts by evaporation of an appropriate solution may yield products similar to those encountered in practice, but the preparation of metal or geological samples spiked with known amounts of impurity is a very different matter, and while it may be easy to remove material from the sample surface (when the impurity has been deposited there by evaporation) it is not necessarily easy to remove material from the interior of a crystallite or from a grain boundary in a fine-grained metal.

\section{CONCLUSIONS}

Though a good deal of work has been done on enrichment procedures, there is scope for further development by systematic planning of separation systems, and much more work needs to be done on investigation of losses by adsorption and on preparation of standards.

\section{REFERENCES}

' C. Liteanu, Mikrochim. Acta, 715 (1970).

2 J. Minczewski, XXth International Congress of Pure and Applied Chemistry, Moscow 1965, Congress Lectures, p. 567, Butterworths: London (1965).

${ }^{3}$ A. Mizuike, 'Separations and Preconcentrations', in Trace Analysis: Physical Methods (Ed. G. H. Morrison), Interscience: New York (1965).

4 J. M. Rottschafer, R. J. Boczkowski and H. B. Mark Jr, Talanta, 19, 163 (1972).

5 T. T. Gorsuch, The Destruction of Organic Matter, Pergamon: Oxford (1970).

${ }^{6}$ H. M. N. H. Irving and W. R. Tomlinson, Talanta, 15, 1267 (1968).

7 G. L. Boorman and W. B. Holbrook, Anal. Chem. 31, 10 (1959).

${ }^{8}$ H. Flaschka, J. Butcher and R. Speights, Talanta, 8, 400 (1961).

9 A. A. Smales and B. D. Pate, Analyst, 77, 188 (1952).

10 T. Y. Toribara, C. P. Shields and L. Koval, Talanta, 17, 1025 (1970).

11 E. Sawicki, Talanta, 16, 1231 (1969).

12 H. Malissa and I. L. Marr, Mikrochim. Acta, 241 (1971).

13 J. Rủžička and J. Starý, Substoichiometry in Radiochemical Analysis, Pergamon: Oxford (1968).

14 G. Tölg, Z. Anal. Chem. 190, 161 (1962).

15 R. A. Chalmers and D. M. Dick, Anal. Chim. Acta, 31, 520 (1964); 32, 117 (1965).

16 R. A. Chalmers and G. Svehla, Solvent Extraction Chemistry, p 600. (Eds. D. Dyrssen, J.-O. Liljenzin and J. Rydberg), North-Holland: Amsterdam (1967).

${ }^{17}$ Yu. A. Zolotov and N. M. Kuz'min, Ekstraktsionnoe kontsentrirovanie (in Russian), Izdat. 'Khimia': Moscow (1971).

18 A. Mizuike, K. Fukuda and Y. Ochiai, Talanta, 19, 527 (1972). 